\title{
From the zero-field metal-insulator transition in two dimensions to the quantum Hall transition: a percolation-effective-medium theory
}

\author{
Yigal Meir \\ Department of Physics, Ben-Gurion University, Beer Sheva 84105, ISRAEL \\ and \\ The Ilse Katz Center for Meso- and Nanoscale Science and Technology, \\ Ben-Gurion University, Beer Sheva 84105, ISRAEL
}

\begin{abstract}
Effective-medium theory is applied to the percolation description of the metal-insulator transition in two dimensions with emphasis on the continuous connection between the zero-magnetic-field transition and the quantum Hall transition. In this model the system consists of puddles connected via saddle points, and there is loss of quantum coherence inside the puddles. The effective conductance of the network is calculated using appropriate integration over the distribution of conductances, leading to a determination of the magnetic field dependence of the critical density. Excellent quantitative agreement is obtained with the experimental data, which allows an estimate of the puddle physical parameters.
\end{abstract}

PACS numbers: 71.30.+h,73.40.Hm,72.15.Rn,73.50.-h

Extensive experimental and theoretical effort has been invested in the attempt to understand and characterize the observation of metallic-like behavior in two dimensions [1]. One of the intriguing experimental findings [2] is that this zero-magnetic-field "transition" is continuously connected with the integer quantum Hall transition. In previous publications [3] I presented a simple non-interacting electron model, combining local quantum transport and global classical percolation, which treated the zero field transition and the quantum Hall transition on the same footing. Numerical calculations showed behavior qualitatively similar to that observed experimentally. In the present paper I present an analytic approach, based on the effective-medium theory of percolation [ $⿴$, that allows quantitative comparison with the experiment. This comparison also allows determination of physical properties of the underlying electronic state. The implications on transport in the quantum Hall regime due to this continuous connection to the zero-field transition are discussed and shown to be in agreement with experimental observations in the quantum Hall regime.

The model is based on two assumptions: (a) the potential fluctuations due to the disorder define density puddles, connected via saddle points, or quantum pointcontacts (QPCs), and (b) The electron wavefunction totally dephases in the puddles, i.e. the time the particle spends in the puddle is larger that the dephasing time. Each saddle point is characterized by its critical energy $\epsilon_{c}$, such that the transmission through it is given by $T(\epsilon)=\Theta\left(\epsilon-\epsilon_{c}\right)$, where quantum tunneling has been neglected. Thus the conductance through each QPC is given by the Landauer formula,

$$
\begin{aligned}
G(\mu, T) & =\frac{2 e^{2}}{h} \int d \epsilon\left(-\frac{\partial f_{F D}(\epsilon)}{\partial \epsilon}\right) T(\epsilon) \\
& =\frac{2 e^{2}}{h} \frac{1}{1+\exp \left[\left(\epsilon_{c}-\mu\right) / k T\right]},
\end{aligned}
$$

where $\mu$ is the chemical potential, and $f_{F D}$ is the FermiDirac distribution function.

The system is now composed of classical resistors, where the resistance of each one of them is given by (1), with random QPC energies. Effective medium theory for such random resistor network has been developed many years ago 胿, and it agrees with exact solution of this network on an infinite lattice (Cayley tree). The resulting equation for the effective conductance $\sigma$ of the total network is given by

$$
0=\int d G f(G) \frac{G-\sigma}{G+(z-2) \sigma},
$$

where $f(G)$ is the distribution function of the conductances in the network, and $z$ is the coordination number of the lattice.

In the present case, the conductance through a point contact, given by (11), depends on the the threshold energy of the quantum point contact and the chemical potential in the puddles that are connected by it. At zero temperature it reduces to $G(\mu, T=0)=2 e^{2} / h \times \Theta(\mu-$ $\left.\epsilon_{c}\right)$, where $\Theta(x)$ is the Heaviside step function. For an arbitrary distribution of the threshold energies $f_{t h r}\left(\epsilon_{c}\right)$, the equation for the effective conductance of the network reads

$$
0=I_{f} \frac{1-\sigma}{1+(z-2) \sigma}+\left(1-I_{f}\right) \frac{-\sigma}{(z-2) \sigma},
$$

with $I_{f} \equiv \int_{-\infty}^{\mu} f_{t h r}\left(\epsilon_{c}\right) d \epsilon_{c}$. Solving for $\sigma$, one finds

$$
\sigma(\mu)=\sigma_{0} \times\left(\mu-\mu_{c}\right)
$$

with $\sigma_{0}$ (of the the order of $e^{2} / h$ ) and $\mu_{c}$ nonuniversal constants, depending on the distribution function and the lattice. The conductance vanishes below a critical chemical potential (density) and grows linearly above it. 
The mean-field critical conductance exponent was found to be unity (compared to $\simeq 1.3$ in two dimensions).

In the presence of a finite perpendicular magnetic field $B$ the situation becomes more complicated. Landau levels form in the puddles (see inset to Fig. 1), and the chemical potential in each puddle oscillates with magnetic field, as it is stuck in the highest occupied Landau level in the puddle. If a puddle is modeled by potential parabolic well, characterized by it depth, $\epsilon_{0}$, and by its confining energy, $\hbar \omega_{0}$ (which also defines its characteristic size), then the chemical potential is given by

$\mu\left(\mu_{0}, \epsilon_{0}, \omega_{0}, B\right)=\epsilon_{0}+\hbar \omega / 2+\hbar \omega\left[\frac{\mu_{0}-\hbar \omega_{0} / 2-\epsilon_{0}}{\hbar \omega}\right]_{i n t}$,

where [ $]_{\text {int }}$ denotes the integer part, $\omega \equiv \sqrt{\omega_{0}^{2}+\omega_{c}^{2}}$, and $w_{c}(B)$ is the cyclotron energy. The parameter $\mu_{0}$ is the zero-field chemical potential (density). For large field the chemical potential is $\epsilon_{0}+\hbar \omega / 2$, the energy of the lowest Landau level. As magnetic field is reduced, there will be a first jump in the chemical potential for $\mu_{0}-\hbar \omega_{0} / 2-\epsilon_{0} \simeq \hbar \omega$, which defines filling factor $\nu=1$. In Fig. 1 a typical magnetic field dependence of the chemical potential in two puddles is depicted. The two puddles differ only in the value of $\epsilon_{0}$, the depth of the potential.

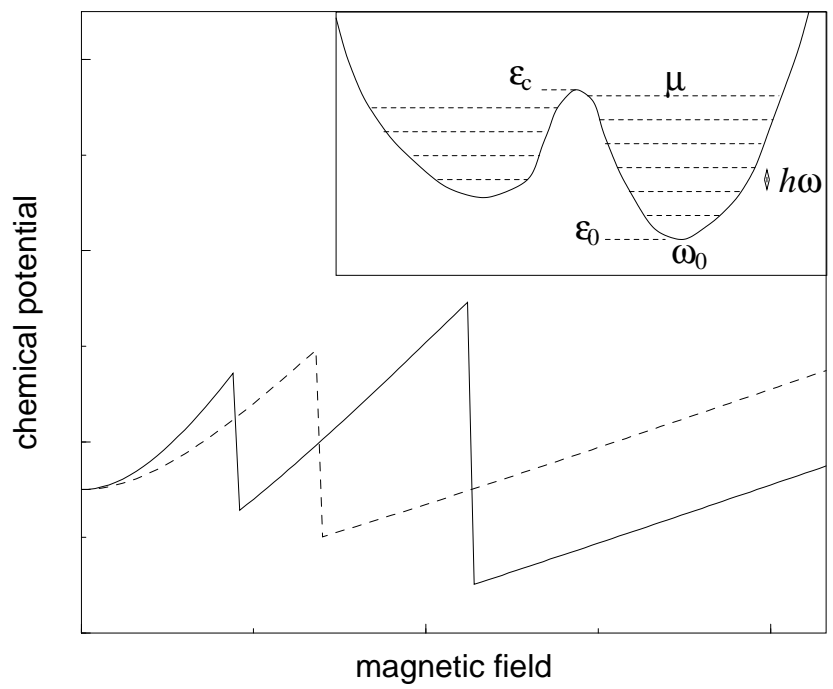

Fig. 1: The magnetic field dependence of the chemical potential in two different puddles. The chemical potential is stuck to the topmost occupied Landau level, which leads to the observed oscillations. The two puddles differ only in their depth. Inset: the parameters characterizing a puddle and a neighboring point contact.

Transport through a single QPC in perpendicular field has been studied experimentally in detail |57. In accordance with the above picture, one finds that the critical gate voltage (density) oscillates with magnetic field due to the depopulation of Landau levels.

The system consists of many puddles, each having its own characteristic physical parameters. Consequently, the integral over the distribution of the conductances in (2), which depends on the distribution of the local puddle parameters through the local chemical potential, $\mu\left(\mu_{0}, \epsilon_{0}, \omega_{0}, B\right)$, has to appropriately performed. Then Eq.(4) turns into

$$
\begin{gathered}
\sigma\left(B, \mu_{0}\right)=\sigma_{0} \times\left[\bar{\mu}\left(\mu_{0}, B\right)-\mu_{c}\right], \quad \text { with } \\
\bar{\mu}\left(\mu_{0}, B\right)= \\
\int d \epsilon_{0}^{\prime} f_{\epsilon_{0}}\left(\epsilon_{0}^{\prime}\right) \int d \omega_{0}^{\prime} f_{\omega_{0}}\left(\omega_{0}^{\prime}\right)\left[\mu\left(\mu_{0}, \epsilon_{0}, \omega_{0}, B\right)-\hbar \omega / 2\right],
\end{gathered}
$$

where $f_{\epsilon_{0}}$ and $f_{\omega_{0}}$ are the distribution functions of the respective puddle parameters. In the following these were assumed to be uniform distributions around characteristic energy scales $\epsilon_{0}$ and $\omega_{0}$, respectively, with respective widths $\Delta \epsilon_{0}$ and $\Delta \omega_{0}$. The energy of the lowest Landau level, $\hbar \omega / 2$, was subtracted, as it does not contribute to the kinetic energy of the electrons (or holes) [6].

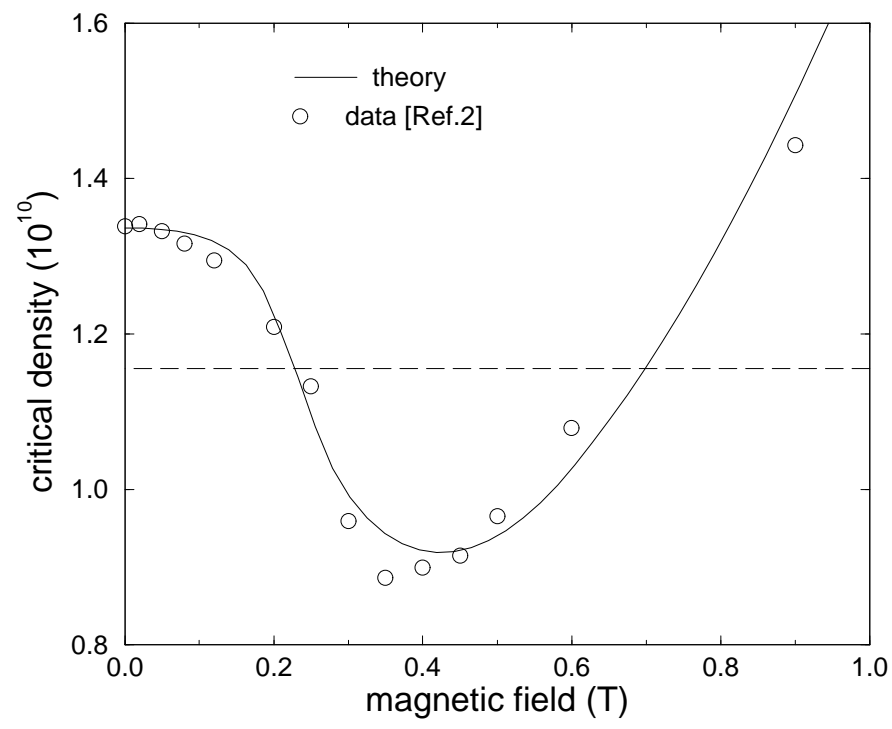

Fig. 2: Comparison of the experimental data [2] for the critical density to the prediction of effective medium theory. The excellent agreement between theory and experiment allows determination of the puddle potential distribution (see text). Note that if the magnetic field is changed for a fixed density (e.g. $n=1.15 \times 10^{10}$ along the broken line), there will be a finite region of magnetic fields where conductance, in the zero-temperature limit, will be non-zero, in contradiction with the quantum-phase-transition description of the quantum Hall effect.

The magnetic field dependence of the critical density $\mu_{0}(B)$ is now determined by the relation 


$$
\bar{\mu}\left(\mu_{0}, B\right)=\mu_{c} .
$$

For large fields $(\nu \leq 1)$ the chemical potential in each puddle varies linearly with magnetic field (see Fig. 1). Thus it is clear that the averaged chemical potential will also vary linearly with field at large fields. As the field is reduced there will be a discontinuous jump in the chemical potential of each puddle at it respective integer filling factors. One might expect that after averaging only the largest jump, at $\nu=1$, may survive, and will be replaced by a smooth rapid increase. This has indeed been seen experimentally [2]. Fig. 2 depicts the experimental data, compared to the predictions of the effective-medium theory, Eqs. (6.7). The parameters $\epsilon_{0}$ and $\omega_{0}$ determine the value of the magnetic field where the rapid increase in the critical density occurs (around $\nu=1$ ), and the saturation value at zero magnetic field. The width of the puddle potential distribution $\Delta \omega_{0}$ determines the rate of increase of the critical density near $\nu=1$. The excellent agreement between the experimental data and theory is evident, and allows a determination of the physical characteristics of the puddle distribution. One finds that the typical confining energy in the puddle is $0.5 \mathrm{meV}$, (with about $10 \%$ dispersion), leading to a typical puddle size of $25 \mathrm{~nm}$.

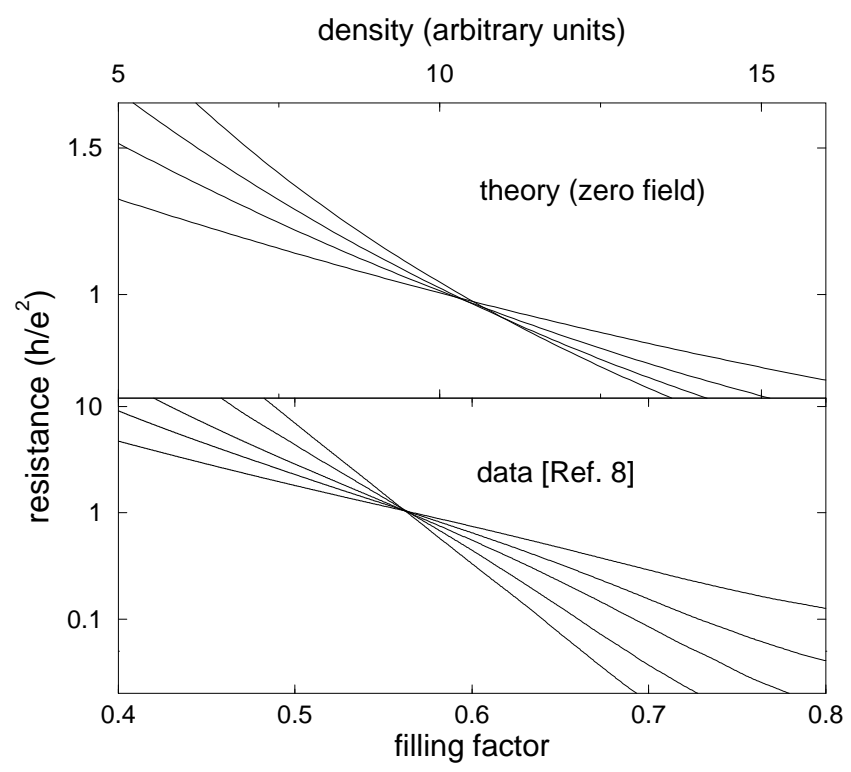

Fig. 3: The exponential dependence of the resistance on density, experiment 8 vs. theory (at zero field). The magnetic field, according to the theory only shifts the transition. As pointed out in [3], the "temperature independent point" in the model has nothing to do with the critical point, which, in this case, is $n=1.05$, way off the scale. Thus all the region depicted in the figure is on the metallic side, which might explain why the Hall resistance remains quantized, as the density crosses this "temperature independent point" 15.

The experimentally demonstrated connection between the quantum Hall transition and the zero-field transition indicates that both stem from the same physical process. The fact that the quantum Hall transition, at least in these systems, is driven by percolation-like behavior and cannot be described by the usual theory of quantum phase transition is demonstrated by the fact that the experimental data in Fig. 2 shows that for as the magnetic field changes a fixed density, $n$ (e.g. $n=1.15 \times 10^{10}$ along the broken line in the figure), there is a finite (and substantial) range in magnetic fields, where the zerotemperature limit of the conductance remains nonzero. This is in contradiction with the usual theory of quantum phase transition that predicts, at zero temperature, a single point where transport is dissipative. Such data has already been reported in the past [7], and the density and temperature dependence of the longitudinal resistance was well described by the relation $\rho_{x x}(n, T)=$ $\rho_{1} \exp \left[\left(n-n_{c}\right) /(\alpha T+\beta)\right]$, where $n_{c}$ is the critical density and $\rho_{1}, \alpha$ and $\beta$ some constants (note the finite resistivity when extrapolated to zero temperature). This behavior looks remarkably like the one reported for the zero-field transition [8], $\rho(n, T)=\rho_{0}+\rho_{1} \exp \left[\left(n-n_{c}\right) /(\alpha T)\right.$. In order to check this functional dependence, the effectivemedium equation (2) was solved for finite temperatures, where a uniform distribution of critical energies $\epsilon_{c}$ was assumed for simplicity. The resulting effective resistance is depicted in Fig. 3, and compared to the experimental observation in the quantum Hall regime [8]. Similarly to the experimental data, the theory shows that there is a wide range in temperature (a high-temperature regime in the theory) where the effective resistance displays exponential temperature dependence [9].

The fact that the quantum Hall transition, at least in some of the reported observations, stems from the same mechanism that leads to the metallic behavior in zero magnetic field is further supported by the fact that the reported current-voltage duality across the quantum Hall transition [10] was also reported for the zero-field transition [11]. The relevance of percolation to the quantum Hall transition has already been established experimentally [12], where it was shown that the transition occurs when the metallic phase percolates through the system, and not at a fixed filling factor, as expected from the quantum-phase-transition scenario.

The relevance of the underlying puddle structure and the finite dephasing rate to these observations in the quantum Hall regime has already been pointed out and elaborated upon by Shimshoni and coworkers 13]. Also, Pryadko and Auerbach have claimed [14] that the finite quantized value of the Hall conductance reported in the insulating regime [15] can only be explained when finite dephasing is taken into account, and the Hall resistance should be infinite in a truly quantum coherent Hall insulator. It should be noted though, that as pointed out in [3] the identification of the "temperature independent point" with the critical point is questionable, and within the model it lies well within the metallic phase. In this picture the fact that the Hall conductance remains quantized through the "transition" is a trivial issue, as this 
point is not the true transition point to the insulator. For the parameters corresponding to the curves depicted in Fig. 3, the critical point occurs at $n=1.05$, thus all the points in the figure lie deep in the metallic phase, and no change in the quantized Hall resistance is expected.

To conclude, the excellent agreement with the experimental data, in addition to previously demonstrated quantitative agreement with temperature and parallel magnetic field data is a further validation of the relevance of the theory to the description of this phenomenon. The theory further predicts that when the conductance is measured in a mesoscopic piece of the system, the conductance will show abrupt changes, as a function of the density, as point contacts open with increasing density. Such mesoscopic jumps in the conductance in the quantum Hall regime have indeed recently observed [16].

The author would like to thank D. Shahar and his group for valuable discussions and for making their data available to him. This work was supported by the Israel Science Foundation - Centers of Excellence Program, and by the German Ministry of Science.

[1] For a recent review of experimental data and theoretical approaches, see E. Abrahams, S. V. Kravchenko and
M. P. Sarachik, cond-mat/0006055.

[2] Y. Hanein et al., Nature 400, 735 (1999).

[3] Y. Meir, Phys. Rev. Lett. 83, 3506 (1999); Phys. Rev. B 61, 16470 (2000).

[4] S. Kirkpatrick, Phys. Rev. Lett. 25, 1722 (1971); R. B. Stinchcombe, J. Phys. C 7, 179 (1974).

[5] See, e.g., B. J. van Wees, Phys. Rev. B43, 12431 (1991).

[6] H. A. Fertig and B. I. Haleprin, Phys. Rev. B 36, 7969 (1987).

[7] M. Hilke et al., Phys. Rev. B 56, R15545 (1997); D. Shahar et al., Solid State Comm 107, 19 (1998).

[8] V. M. Pudalov, JETP Lett. 66, 175 (1997); Y. Hanein et al., Phys. Rev. Lett. 80, 1288 (1998).

[9] It should be noted, though, that the resistance change in the data is much larger than that in the theory.

[10] D. Shahar et al., Science 274, 589 (1996); E. Shimshoni et al., Phys. Rev. B 55, 13730 (1996).

[11] D. Simonian, S. V Kravchenko and M. P. Sarachik, Phys. Rev. B 55, R13421 (1997).

[12] A. A. Shashkin et al., Phys. Rev. Lett. 73, 3141 (1994); V. T. Dolgapolov et al., JETP Lett. 62, 162 (1995); I. V. Kukuskin et al., Phys. Rev. B53, R13260 (1996).

[13] E. Shimshoni and A. Auerbach, Phys. Rev. B 55, 9817 (1997); E. Shimshoni, A. Auerbach and A. Kapitulnik, Phys. Rev. Lett. 80, 3352 (1998). E. Shimsoni, Phys. Rev. B 60, 10691 (1999)

[14] L. P. Pryadko and A. Auerbach, Phys. Rev. Lett. 82, 1253 (1999).

[15] M. Hilke et al., Nature 395, 675 (1998).

[16] D. Shahar, unpublished. 